\title{
Quantum periodic cluster methods for strongly correlated electron systems
}

\author{
Tran Minh-Tien \\ Asia Pacific Center for Theoretical Physics, POSTECH, Pohang, Republic of Korea, \\ Institute of Physics and Electronics, Vietnamese Academy of Science and Technology, Hanoi, Vietnam.
}

\begin{abstract}
Quantum periodic cluster methods for strongly correlated electron systems are reformulated and developed. The reformulation and development are based on a canonical transformation which periodizes the fermions in the cluster space. The dynamical cluster approximation and the cellular dynamical mean field theory are related each other through the canonical transformation. A cluster perturbation theory with periodic boundary conditions is developed. It is found that the periodic cluster perturbation theory converges rapidly with corrections $\mathcal{O}\left(1 / L_{c}^{2}\right)$, where $L_{c}$ is the linear size of the clusters, whereas the ordinary cluster perturbation theory converges with corrections $\mathcal{O}\left(1 / L_{c}\right)$.
\end{abstract}

PACS numbers: 71.10.-w, 71.10.Fd, 71.27.+a, 71.10.Pm

\section{INTRODUCTION}

One of the most active areas in the condensed matter physics is the search for new methods which could capture essential features of electron correlations and disorder in a controllable manner. Perhaps the most successful and widely used methods are the dynamical mean field theory (DMFT) ${ }^{1.2}$ and the coherent potential approximation (CPA) 3.4 .5 Both these methods are exact in infinite dimensions. However, at finite dimensions they neglect nonlocal correlations. This limitation has raised interests in developing of quantum cluster methods which could capture nonlocal correlations. The most successful of self consistent quantum cluster methods are the molecular coherent potential approximation (MCPA) for disorder systems, $\underline{\underline{6}}$ and the cellular dynamical mean field theory (CDMFT $)^{7}$ and the dynamical cluster approximation (DCA $)^{8.9}$ for correlated electron systems. In these quantum cluster theories the system lattice is split into a series of clusters. Both local and nonlocal correlations within the clusters are treated exactly, whereas the nonlocal correlations between different clusters are treated in a mean-field approximation. The mean field is taken into account by mapping the lattice problem onto a self-consistent effective cluster problem. The MCPA and CDMFT are formulated on the real space, and share a common microscopic definition. Within the CDMFT (or the MCPA) the cluster Green function is calculated with open boundary conditions. The DCA is traditionally formulated in the reciprocal space. It is based on the idea of discretizing irreducible quantities on the reciprocal space. Within the DCA the cluster Green function is calculated with periodic boundary conditions. Due to the difference of boundary conditions it seems that the DCA and the CDMFT (or the MCPA) have different microscopic definitions. For instance, the CDMFT can be formulated within the self-energy functional approach, whereas it seems that the DCA cannot be ${ }^{10,11}$ There is also a view of the DCA in the real space representation which shows a relation between the DCA and CDMFT 12.13.14 The view is based on an unitary transformation of the hopping matrix. However, the interaction part still remains unchanged. In this paper we show that the DCA and the CDMFT share a common microscopic definition through a canonical transformation. The canonical transformation is similar to the unitary transformation which was used to view the DCA in the real space. It periodizes the fermions in the cluster space, that the cluster Green function has periodic boundary conditions. We apply the canonical transformation to the whole Hamiltonian, and in the presence of a constrain which prevents the umklapp momentum transfer from the superlattice to the cluster space we obtain a periodized Hamiltonian. The umklapp momentum transfer appears when the sum of two momenta of the superlattice is beyond the first Brillouin zone, and is solely due to the imaginary superlattice construction of clusters with periodic boundary conditions. Within the periodized Hamiltonian the DCA can be derived from the CDMFT. In the such way, the DCA and the CDMFT can be unified into a common microscopic background. The derivation also gives the microscopic background of the discretization of irreducible quantities on the reciprocal space, and clarifies the approximation nature of the DCA.

The present paper consists of two parts. In the first part a derivation of the DCA from the CDMFT through the canonical transformation for fermions is presented. The second part is concerned with the cluster perturbation theory (CPT) ${ }^{15.16 .17}$ The CPT is also a quantum cluster approach. However, in difference with the CDMFT or the DCA, the CPT does not have the self consistency. The CPT can be viewed as the first term of a systematic expansion around strong coupling. However, within the CPT the cluster Green function is calculated with open boundary conditions, that the wave vector within a cluster is no longer a conserved quantum number. There are also several approaches which generate periodic boundary conditions for the CPT. One is to add appropriate hopping terms and then subtract them within strong coupling perturbation theory 18 However, this approach give results which are less accurate than the ones from open boundary conditions ${ }^{17}$ The other approach adapts the periodicity of the clusters from the DCA. It was used to study the Ising model ${ }^{\frac{9}{\underline{a}}}$ In this paper we present the periodic cluster perturbation theory 
for fermion systems. The periodic cluster perturbation theory (PCPT) is the CPT formulated for the periodized fermions which are obtained after making the canonical transformation. The PCPT has truly the small parameter $1 / L_{c}$, where $L_{c}$ is the cluster linear size. It turns out that the PCPT converges quadratically in $1 / L_{c}$, whereas the CPT converges linearly in $1 / L_{c}$. This is also tested on the study of an exact solvable model.

The plan of the present paper is as follows. In Sec. II we present the canonical transformation which transforms the original fermions onto periodized ones. In Sec. III we derive the DCA from the CDMFT after making the canonical transformation. The PCPT is presented in Sec. IV. In this section we also study an exact solvable model in both the direct and periodized forms. Finally, the conclusion and remarks are presented in Sec. V.

\section{PERIODIZED FERMIONS}

We present a periodization for fermions through a canonical transformation of fermionic operators. For an illustration a fairly general model of correlated electron systems is considered. The Hamiltonian of the model reads

$$
\begin{aligned}
H= & \sum_{i, j, \sigma} t(i, j) c_{i \sigma}^{\dagger} c_{j \sigma} \\
& +\sum_{i, j} \sum_{\{\sigma\}} U^{\{\sigma\}}(i, j) c_{i \sigma_{1}}^{\dagger} c_{i \sigma_{2}} c_{j \sigma_{3}}^{\dagger} c_{j \sigma_{4}}
\end{aligned}
$$

where $\{\sigma\}=\left\{\sigma_{1}, \sigma_{2}, \sigma_{3}, \sigma_{4}\right\} . \quad c_{i \sigma}^{\dagger}\left(c_{i \sigma}\right)$ is the creation (annihilation) operator for fermion with spin $\sigma$ at lattice site $i . t(i, j)$ is the hopping integral, and $U^{\{\sigma\}}(i, j)$ is the interaction strength. We will consider a hypercubic lattice of linear size $L$ with the lattice constant $a=1$ on $d$ dimensions. The number of lattice sites thus is $N=L^{d}$. The lattice is divided into a set of identical clusters of linear size $L_{c}$. The number of lattice sites inside a cluster thus is $N_{c}=L_{c}^{d}$. The set of the clusters form a superlattice. We use letters $i, j, \ldots$ to label the lattice site of the original lattice, and use $\mathbf{r}_{i}$ to denote the position of site $i$. Letters $\mathbf{k}, \mathbf{p}, \ldots$ are used to denote the wave vectors of the reciprocal space of the original lattice. Capital letters $I, J, \ldots$ are used to label the origin of the clusters. The origin coordinate of clusters are denoted by $\mathbf{R}_{I}$, and the wave vectors of the reciprocal space of the superlattice are denoted by $\mathbf{K}, \mathbf{P}, \ldots$ The lattice sites inside a cluster are labeled by $a, b, \ldots$ Their position is denoted by $\overline{\mathbf{r}}_{a}, \overline{\mathbf{r}}_{b}, \ldots$ and the wave vectors of the reciprocal space of the cluster are denoted by $\overline{\mathbf{k}}, \overline{\mathbf{p}}, \ldots$ Thus $\mathbf{r}_{i}=\mathbf{R}_{I}+\overline{\mathbf{r}}_{a}, \mathbf{k}=\mathbf{K}+\overline{\mathbf{k}}, \ldots$ Note that $\exp (i \overline{\mathbf{k}} \cdot \mathbf{R})=1$, since $\bar{k}_{\alpha}=2 \pi n_{\alpha} / L_{c}$, and $R_{\alpha}=m_{\alpha} L_{c}$, where $n_{\alpha}, m_{\alpha}$ are integer. We use the Greek letter $\sigma$ to denote the spin (or other quantum) variables. With these notations we rewrite the operators and model parameters as $c_{i \sigma} \equiv c_{a \sigma}(I), t(i, j) \equiv t_{a b}(I, J)$, $U^{\{\sigma\}}(i, j) \equiv U_{a b}^{\{\sigma\}}(I, J), \ldots$ Denoting $c_{\mathbf{k} \sigma}^{\dagger}, c_{\mathbf{k} \sigma}$ the Fourier transform of $c_{i \sigma}^{\dagger}, c_{i \sigma}$, the Hamiltonian (11) can be rewritten in the reciprocal space representation

$$
\begin{aligned}
H= & \sum_{\mathbf{k}, \sigma} t(\mathbf{k}) c_{\mathbf{k} \sigma}^{\dagger} c_{\mathbf{k} \sigma} \\
& +\frac{1}{N} \sum_{\mathbf{k}, \mathbf{k}^{\prime}, \mathbf{p}} \sum_{\{\sigma\}} U^{\{\sigma\}}(\mathbf{p}) c_{\mathbf{k}+\mathbf{p}, \sigma_{1}}^{\dagger} c_{\mathbf{k}, \sigma_{2}} c_{\mathbf{k}^{\prime}-\mathbf{p}, \sigma_{3}}^{\dagger} c_{\mathbf{k}^{\prime}, \sigma_{4}},
\end{aligned}
$$

where $t(\mathbf{k})$ and $U^{\{\sigma\}}(\mathbf{p})$ are the Fourier transformation of $t(i, j)$ and $U^{\{\sigma\}}(i, j)$, respectively, i.e.,

$$
\begin{aligned}
t(i, j) & =\frac{1}{N} \sum_{\mathbf{k}} t(\mathbf{k}) e^{i \mathbf{k} \cdot\left(\mathbf{r}_{i}-\mathbf{r}_{j}\right)} \\
U^{\{\sigma\}}(i, j) & =\frac{1}{N} \sum_{\mathbf{k}} U^{\{\sigma\}}(\mathbf{k}) e^{i \mathbf{k} \cdot\left(\mathbf{r}_{i}-\mathbf{r}_{j}\right)} .
\end{aligned}
$$

We introduce operators

$$
\begin{aligned}
& \bar{c}_{a \sigma}(\mathbf{K})=\sqrt{\frac{N_{c}}{N}} \sum_{I} c_{a \sigma}(I) e^{-i \mathbf{K} \cdot\left(\mathbf{R}_{I}+\overline{\mathbf{r}}_{a}\right)}, \\
& \bar{c}_{a \sigma}^{\dagger}(\mathbf{K})=\sqrt{\frac{N_{c}}{N}} \sum_{I} c_{a \sigma}^{\dagger}(I) e^{i \mathbf{K} \cdot\left(\mathbf{R}_{I}+\overline{\mathbf{r}}_{a}\right)} .
\end{aligned}
$$

One can check that these operators satisfy the anticommutation relations

$$
\begin{aligned}
& \left\{\bar{c}_{a \sigma}(\mathbf{K}), \bar{c}_{b \sigma^{\prime}}^{\dagger}(\mathbf{P})\right\}=\delta_{a b} \delta_{\sigma \sigma^{\prime}} \delta(\mathbf{K}-\mathbf{P}) \\
& \left\{\bar{c}_{a \sigma}(\mathbf{K}), \bar{c}_{b \sigma^{\prime}}(\mathbf{P})\right\}=0 \\
& \left\{\bar{c}_{a \sigma}^{\dagger}(\mathbf{K}), \bar{c}_{b \sigma^{\prime}}^{\dagger}(\mathbf{P})\right\}=0
\end{aligned}
$$

where $\{A, B\}=A B+B A$. Since the anticommutation relations are preserved, the transformation (5)-(6) is canonical. The inverse transformation reads

$$
\begin{aligned}
c_{a \sigma}(I) & =\sqrt{\frac{N_{c}}{N}} \sum_{\mathbf{K}} \bar{c}_{a \sigma}(\mathbf{K}) e^{i \mathbf{K} \cdot\left(\mathbf{R}_{I}+\overline{\mathbf{r}}_{a}\right)}, \\
c_{a \sigma}^{\dagger}(I) & =\sqrt{\frac{N_{c}}{N}} \sum_{\mathbf{K}} \bar{c}_{a \sigma}^{\dagger}(\mathbf{K}) e^{-i \mathbf{K} \cdot\left(\mathbf{R}_{I}+\overline{\mathbf{r}}_{a}\right)} .
\end{aligned}
$$

We also introduce other operators in the real superlattice space

$$
\begin{aligned}
& \bar{c}_{a \sigma}(I)=\sqrt{\frac{N_{c}}{N}} \sum_{\mathbf{K}} \bar{c}_{a \sigma}(\mathbf{K}) e^{i \mathbf{K} \cdot \mathbf{R}_{I}}, \\
& \bar{c}_{a \sigma}^{\dagger}(I)=\sqrt{\frac{N_{c}}{N}} \sum_{\mathbf{K}} \bar{c}_{a \sigma}^{\dagger}(\mathbf{K}) e^{-i \mathbf{K} \cdot \mathbf{R}_{I}} .
\end{aligned}
$$

One can check that these operators (9)-(10) also satisfy the fermionic anticommutation relations. They annihilate or create a fermion at site $(I, a)$. The transformation (9) - (10) can be viewed as the Fourier transformation for the fermion operators $\bar{c}_{a \sigma}(\mathbf{K}), \bar{c}_{a \sigma}^{\dagger}(\mathbf{K})$ in the superlattice 
space. From Eqs. (9)-(10) and (5)-(6), one can show that

$$
\begin{aligned}
\bar{c}_{a \sigma}(I) & =\sqrt{\frac{1}{N}} \sum_{\mathbf{K}, \overline{\mathbf{k}}} c_{\mathbf{K}+\overline{\mathbf{k}}, \sigma} e^{i \mathbf{K} \cdot \mathbf{R}_{I}+i \overline{\mathbf{k}} \cdot \overline{\mathbf{r}}_{a}}, \\
\bar{c}_{a \sigma}^{\dagger}(I) & =\sqrt{\frac{1}{N}} \sum_{\mathbf{K}, \overline{\mathbf{k}}} c_{\mathbf{K}+\overline{\mathbf{k}}, \sigma}^{\dagger} e^{-i \mathbf{K} \cdot \mathbf{R}_{I}-i \overline{\mathbf{k}} \cdot \overline{\mathbf{r}}_{a}} .
\end{aligned}
$$

In deriving Eqs. (11)-(12) we have used $\exp \left(i \overline{\mathbf{k}} \cdot \mathbf{R}_{I}\right)=1$. Since $\exp \left(i \overline{\mathbf{k}} \cdot \overline{\mathbf{r}}_{a+L_{c}}\right)=\exp \left(i \overline{\mathbf{k}} \cdot \overline{\mathbf{r}}_{a}\right)$, one can see that the fermionic operators $\bar{c}_{a \sigma}(I), \bar{c}_{a \sigma}^{\dagger}(I)$ are periodic in the indexes $a$, whereas the original ones $c_{a \sigma}(I), c_{a \sigma}^{\dagger}(I)$ are not. The fermion created by $\bar{c}_{a \sigma}^{\dagger}(I)$ is periodic in both the superlattice and cluster spaces. Due to the periodic property we call $\bar{c}_{a \sigma}(I), \bar{c}_{a \sigma}^{\dagger}(I)$ the periodized fermion operators. The periodicity allows to perform the Fourier transformation of $\bar{c}_{a \sigma}(I), \bar{c}_{a \sigma}^{\dagger}(I)$ in the superlattice and cluster spaces

$$
\begin{aligned}
& \bar{c}_{\overline{\mathbf{k}} \sigma}(\mathbf{K})=\sqrt{\frac{1}{N}} \sum_{I, a} \bar{c}_{a \sigma}(I) e^{-i \mathbf{K} \cdot \mathbf{R}_{I}-i \overline{\mathbf{k}} \cdot \overline{\mathbf{r}}_{a}} \\
& \bar{c}_{\overline{\mathbf{k}} \sigma}^{\dagger}(\mathbf{K})=\sqrt{\frac{1}{N}} \sum_{I, a} \bar{c}_{a \sigma}^{\dagger}(I) e^{i \mathbf{K} \cdot \mathbf{R}_{I}+i \overline{\mathbf{k}} \cdot \overline{\mathbf{r}}_{a}}
\end{aligned}
$$

We want to emphasise here that both $\mathbf{K}$ and $\overline{\mathbf{k}}$ should be restricted to the first Brillouin zones of the superlattice and cluster spaces. From Eqs. (11)-(12) we obtain immediately $\bar{c}_{\overline{\mathbf{k}} \sigma}(\mathbf{K})=c_{\overline{\mathbf{k}}+\mathbf{K}, \sigma}, \bar{c}_{\overline{\mathbf{k}} \sigma}^{\dagger}(\mathbf{K})=c_{\overline{\mathbf{k}}+\mathbf{K}, \sigma}^{\dagger}$. However, these relations are valid only for momenta in the first Brillouin zones. Thus, from the Hamiltonian (2) we obtain

$$
\begin{aligned}
H= & \sum_{\mathbf{K}, \overline{\mathbf{k}}, \sigma} t(\mathbf{K}+\overline{\mathbf{k}}) \bar{c}_{\overline{\mathbf{k}} \sigma}^{\dagger}(\mathbf{K}) \bar{c}_{\overline{\mathbf{k}} \sigma}(\mathbf{K}) \\
& +\frac{1}{N} \sum_{\mathbf{K}, \mathbf{K}^{\prime}, \mathbf{P}} \sum_{\overline{\mathbf{k}}, \overline{\mathbf{k}}^{\prime}, \overline{\mathbf{p}}} \sum_{\{\sigma\}} U^{\{\sigma\}}(\mathbf{P}+\overline{\mathbf{p}}) \bar{c}_{\overline{\mathbf{k}}+\overline{\mathbf{p}}, \sigma_{1}}^{\dagger}(\mathbf{K}+\mathbf{P}) \\
& \bar{c}_{\overline{\mathbf{k}}, \sigma_{2}}(\mathbf{K}) \bar{c}_{\overline{\mathbf{k}}^{\prime}-\overline{\mathbf{p}}, \sigma_{3}}^{\dagger}\left(\mathbf{K}^{\prime}-\mathbf{P}\right) \bar{c}_{\overline{\mathbf{k}}^{\prime}, \sigma_{4}}\left(\mathbf{K}^{\prime}\right) .
\end{aligned}
$$

Note that in Hamiltonian (15) all sums over momenta are restricted to the first Brillouin zones. Moreover, when $\mathbf{K}+\mathbf{P}$ and $\mathbf{K}^{\prime}-\mathbf{P}$ are beyond the first Brillouin zone, one has to translate them back to the first Brillouin zone, thus the momentum sums still are over the full first Brillouin zone. Equivalently, the momenta of the periodized fermion operators take values $K_{\alpha}=$ $2 \pi \bmod \left(n_{\alpha}, L / L_{c}\right) / L-\pi / L_{c}$. In deriving the Hamiltonian (15) we have used the following restriction

$$
\begin{aligned}
\delta(\mathbf{p}+\mathbf{k}-\mathbf{q}) & \equiv \delta(\mathbf{P}+\mathbf{K}-\mathbf{Q}+\overline{\mathbf{G}}) \delta\left(\overline{\mathbf{p}}+\overline{\mathbf{k}}-\overline{\mathbf{q}}+\overline{\mathbf{G}}^{\prime}\right) \\
& =\delta(\mathbf{P}+\mathbf{K}-\mathbf{Q}+\overline{\mathbf{G}}) \delta(\overline{\mathbf{p}}+\overline{\mathbf{k}}-\overline{\mathbf{q}}), \quad(16)
\end{aligned}
$$

where $\overline{\mathbf{G}}, \overline{\mathbf{G}}^{\prime}$ are arbitrary momenta which are either zero or a vector of the reciprocal superlattice space. $\overline{\mathbf{G}}$, $\overline{\mathbf{G}}^{\prime}$ are also the momenta of the reciprocal cluster space.
$\overline{\mathbf{G}} \neq 0$ just means an umklapp process on the superlattice, and $\overline{\mathbf{G}}^{\prime}$ can be interpreted as a momentum transfered by the umklapp process from the superlattice to the cluster space. The first line of Eq. (16) is exact, while the second line is a particular restriction. This restriction prevents the momentum transfer in the umklapp processes of the superlattice. The umklapp processes appear when the sum of two momenta of the superlattice is beyond the first Brillouin zone, and they transfer a momentum modulo a vector of the reciprocal superlattice to the reciprocal cluster space that generate a momentum nonconservation. However, the such momentum transfer is unphysical, it is solely due to the imaginary discretization of the original lattice into superlattice of clusters with periodic boundary conditions. In order to maintain the correct physics, one has to impose a constrain which forbids the umklapp momentum transfer on the superlattice. The restriction (16) is the constrain of the superlattice construction. As we will see later on an example of exact solvable model, the constrain indeed leads the periodized Hamiltonian (15) to the exact solution. Performing the Fourier transformation (13)-(14), from the Hamiltonian (15) we obtain

$$
\begin{aligned}
& H=\sum_{I, J, a, b, \sigma} \bar{t}_{a b}(I, J) \bar{c}_{a \sigma}^{\dagger}(I) \bar{c}_{b \sigma}(J)+ \\
& \sum_{I, J, a, b,\{\sigma\}} \bar{U}_{a b}^{\{\sigma\}}(I, J) \bar{c}_{a \sigma_{1}}^{\dagger}(I) \bar{c}_{a \sigma_{2}}(I) \bar{c}_{b \sigma_{3}}^{\dagger}(J) \bar{c}_{b \sigma_{4}}(J),
\end{aligned}
$$

where the hopping integral and interaction of the periodized fermions now are

$$
\begin{gathered}
\bar{t}_{a b}(I, J)=\frac{1}{N} \sum_{\mathbf{K}, \overline{\mathbf{k}}} t(\mathbf{K}+\overline{\mathbf{k}}) e^{i \mathbf{K} \cdot\left(\mathbf{R}_{I}-\mathbf{R}_{J}\right)+i \overline{\mathbf{k}} \cdot\left(\overline{\mathbf{r}}_{a}-\overline{\mathbf{r}}_{b}\right)},(1 \\
\bar{U}_{a b}^{\{\sigma\}}(I, J)=\frac{1}{N} \sum_{\mathbf{K}, \overline{\mathbf{k}}} U^{\{\sigma\}}(\mathbf{K}+\overline{\mathbf{k}}) e^{i \mathbf{K} \cdot\left(\mathbf{R}_{I}-\mathbf{R}_{J}\right)+i \overline{\mathbf{k}} \cdot\left(\overline{\mathbf{r}}_{a}-\overline{\mathbf{r}}_{b}\right)} .
\end{gathered}
$$

One can notice that $\bar{t}_{a b}(I, J), \bar{U}_{a b}^{\{\sigma\}}(I, J)$ are cyclic in the indexes $a, b$, whereas $t_{a b}(I, J), U_{a b}^{\{\sigma\}}(I, J)$ are not. This means that the Fourier transformation of $\bar{t}_{a b}(I, J)$ and $\bar{U}_{a b}^{\{\sigma\}}(I, J)$ are diagonal in the reciprocal space. Denoting $\bar{t}_{\overline{\mathbf{k}}}(\mathbf{K}), \bar{U}_{\overline{\mathbf{k}}}^{\{\sigma\}}(\mathbf{K})$ the Fourier transformation of $\bar{t}_{a b}(I, J)$, $\bar{U}_{a b}^{\{\sigma\}}(I, J)$, one can see immediately from Eqs. (18)-(19) that $\bar{t}_{\overline{\mathbf{k}}}(\mathbf{K})=t(\mathbf{K}+\overline{\mathbf{k}}), \bar{U}_{\overline{\mathbf{k}}}^{\{\sigma\}}(\mathbf{K})=U^{\{\sigma\}}(\mathbf{K}+\overline{\mathbf{k}})$. Note that the hopping term (18) was also obtained previously by employing an unitary transformation. ${ }^{12.14}$ The unitary transformation is similar to the canonical transformation for fermion operators. Beside the periodicity, the periodized hopping (18) and interaction (19) are quite different from the original hopping and interaction, respectively. They may connect those lattice sites that the original ones do not. For instance, if the original hopping is nearest neighbor then the hopping only couples the nearest neighbor sites either within the clusters, or 
on the cluster boundaries of the nearest neighbor clusters. However, in the same case the periodized hopping can couple a lattice site with any other lattice site. The Hamiltonian (17) is adequate to quantum cluster methods with periodic boundary conditions.

Next, we consider the one-particle Green function of the periodized fermions

$$
\bar{G}_{a b}(\mathbf{K}, z)=\ll \bar{c}_{a \sigma}(\mathbf{K}) \mid \bar{c}_{b \sigma}^{\dagger}(\mathbf{K}) \gg_{z}
$$

Without difficulty one can show that

$$
\bar{G}_{a b}(\mathbf{K})=\frac{1}{N_{c}} \sum_{\overline{\mathbf{k}}} G(\mathbf{K}+\overline{\mathbf{k}}) e^{i \overline{\mathbf{k}} \cdot\left(\overline{\mathbf{r}}_{a}-\overline{\mathbf{r}}_{b}\right)},
$$

where $G(\mathbf{k})=\ll c_{\mathbf{k} \sigma} \mid c_{\mathbf{k} \sigma}^{\dagger} \gg_{z}$ is the Green function of the original fermions. Equation (21) shows that the Green function of the periodized fermions is diagonal in the reciprocal cluster space. We immediately obtain

$$
G(\mathbf{K}+\overline{\mathbf{k}}, z)=\bar{G}_{\overline{\mathbf{k}}}(\mathbf{K}, z)
$$

Equation (22) expresses the identity of the Green functions of the original fermions and of the periodized fermions. The Dyson equation of the Green function $\bar{G}_{a b}(\mathbf{K}, z)$ reads

$$
[\hat{G}(\mathbf{K}, z)]^{-1}=z \hat{I}-\hat{\bar{t}}(\mathbf{K})-\hat{\Sigma}(\mathbf{K}, z),
$$

where the hat symbol denotes the matrix notation in the cluster space. $\hat{\Sigma}(\mathbf{K}, z)$ is the self energy. Since the Green function $\hat{G}(\mathbf{K}, z)$ and the hopping $\hat{\bar{t}}(\mathbf{K})$ are diagonal in the reciprocal cluster space, the self energy $\hat{\Sigma}(\mathbf{K}, z)$ must diagonal in the reciprocal cluster space too. This is consistent with the Hamiltonian (17), where both the hopping and interaction terms are diagonal.

\section{DYNAMICAL CLUSTER APPROXIMATION}

With the periodized Hamiltonian (17) we can derive the DCA from the CDMFT (or from the MCPA). Applying the CDMFT to the periodized fermions we obtain

$$
\hat{\bar{G}}(\mathbf{K}, z)=[z \hat{I}-\hat{\bar{t}}(\mathbf{K})-\hat{\bar{\Sigma}}(z)]^{-1}
$$

The hopping matrix $\hat{\bar{t}}(\mathbf{K})$ is the Fourier transformation of the periodized hopping (18) in the superlattice space, i.e.,

$$
\bar{t}_{a b}(\mathbf{K})=\frac{1}{N_{c}} \sum_{\overline{\mathbf{k}}} t(\mathbf{K}+\overline{\mathbf{k}}) e^{i \overline{\mathbf{k}} \cdot\left(\overline{\mathbf{r}}_{a}-\overline{\mathbf{r}}_{b}\right)}
$$

The self energy $\hat{\bar{\Sigma}}(z)$ is determined from an effective single cluster problem. The action of the effective single cluster is

$$
\begin{aligned}
S_{\mathrm{eff}}= & -\int_{0}^{\beta} \int_{0}^{\beta} d \tau d \tau^{\prime} \sum_{a, b, \sigma} \bar{c}_{a \sigma}^{\dagger}(\tau) \overline{\mathcal{G}}_{a b}^{-1}\left(\tau-\tau^{\prime}\right) \bar{c}_{b \sigma}\left(\tau^{\prime}\right) \\
& +\int_{0}^{\beta} d \tau \sum_{a, b,\{\sigma\}} \bar{U}_{a b}^{\{\sigma\}}\left(\bar{c}_{a \sigma_{1}}^{\dagger} \bar{c}_{a \sigma_{2}} \bar{c}_{b \sigma_{3}}^{\dagger} \bar{c}_{b \sigma_{4}}\right)(\tau),
\end{aligned}
$$

where $\overline{\mathcal{G}}_{a b}^{-1}\left(\tau-\tau^{\prime}\right)$ plays the role of the effective mean field acting on the cluster. The interaction in the effective single cluster is obtained from Eq. (19)

$$
\bar{U}_{a b}^{\{\sigma\}}=\frac{1}{N} \sum_{\mathbf{K}, \overline{\mathbf{k}}} U^{\{\sigma\}}(\mathbf{K}+\overline{\mathbf{k}}) e^{i \overline{\mathbf{k}} \cdot\left(\overline{\mathbf{r}}_{a}-\overline{\mathbf{r}}_{b}\right)} .
$$

The self consistency requires the identity of the cluster Green function obtained from the effective single cluster problem (26) and the cluster Green function of the original cluster, i.e.,

$$
\frac{N_{c}}{N} \sum_{\mathbf{K}} \hat{\bar{G}}(\mathbf{K}, z)=\left[\hat{\overline{\mathcal{G}}}^{-1}(z)-\hat{\bar{\Sigma}}(z)\right]^{-1}
$$

Since in the reciprocal cluster space the lattice Green function $\hat{\bar{G}}(\mathbf{K}, z)$ and the self energy $\hat{\bar{\Sigma}}(z)$ are diagonal, the Green function of the effective medium $\hat{\overline{\mathcal{G}}}(z)$ must be diagonal too. After making the Fourier transformation, we obtain from Eqs. (24), (28)

$$
\begin{gathered}
\bar{G}_{\overline{\mathbf{k}}}(\mathbf{K}, z)=\frac{1}{z-\bar{t}_{\overline{\mathbf{k}}}(\mathbf{K})-\bar{\Sigma}_{\overline{\mathbf{k}}}(z)}, \\
\frac{N_{c}}{N} \sum_{\mathbf{K}} \bar{G}_{\overline{\mathbf{k}}}(\mathbf{K}, z)=\frac{1}{\overline{\mathcal{G}}_{\overline{\mathbf{k}}}^{-1}(z)-\bar{\Sigma}_{\overline{\mathbf{k}}}(z)} .
\end{gathered}
$$

Since $\bar{t}_{\overline{\mathbf{k}}}(\mathbf{K})=t(\overline{\mathbf{k}}+\mathbf{K}), \bar{U}_{\overline{\mathbf{k}}}=\left(N_{c} / N\right) \sum_{\mathbf{K}} U(\mathbf{K}+\overline{\mathbf{k}})$, and $\bar{G}_{\overline{\mathbf{k}}}(\mathbf{K}, z)=G(\overline{\mathbf{k}}+\mathbf{K}, z)$ we recover the DCA equations. Equations (24)-(28) can be viewed as the DCA equations formulated in the real cluster space. Originally, the DCA is formulated in the reciprocal space ${ }^{8}$ It is based on the idea of discretizing irreducible quantities on the reciprocal space. Later, the DCA was also viewed in the real space by introducing an unitary transformation for the hopping matrix. ${ }^{12,13,14}$ However, in this view only the hopping term is transformed, whereas the interaction term remains unchanged. As a consequence, the hopping and interaction terms are not treated on an equal footing. It correctly recovers the DCA only for a local single-site interaction. In the presented approach both the hopping and interaction terms are treated on an equal footing. It also clarifies why within the DCA the lattice quantities, for instance the hopping term in the lattice Green function (Eq. (25)), are unchanged, whereas the cluster quantities, for instance the cluster interactions (Eq. (27)), are discretized or coarse grained. Thus, the DCA can be used in both the reciprocal and real spaces. In the real space the DCA is just the CDMFT applied to the periodized Hamiltonian (17). Due to this formulation the 
DCA has the properties of the CDMFT. For instance, the DCA can also be formulated within the self-energy functional approach in the real space by the same way as of the CDMFT ${ }^{10.11}$ So far we have shown that both the DCA and CDMFT share a common microscopic definition. They are related each other through the canonical periodization transformation. An extensive discussion about the two methods is already given in the recent review $\stackrel{9}{\underline{9}}$

\section{PERIODIC CLUSTER PERTURBATION THEORY}

The CPT is also a quantum cluster approach, although it does not have the self consistency ${ }^{15.16 .17}$ In this section we present a CPT with periodic boundary conditions. We refer it as PCPT. Basically, the PCPT is the CPT applied to the periodized fermions. A similar PCPT which employs the formulation of the DCA for spin systems was used previously to study the Ising model .90 will consider the system with the nearest neighbor hopping. In the periodization version the hopping (18) is no longer only nearest neighbor. It may couple a lattice site with any other lattice site. As the CPT, the PCPT splits the intra- and inter-cluster parts of the hopping term. The intra-cluster part of the Hamiltonian is

$$
H^{\mathrm{c}}=\sum_{a, b, \sigma} \bar{t}_{a b} \bar{c}_{a \sigma}^{\dagger} \bar{c}_{b \sigma}+\sum_{a, b,\{\sigma\}} \bar{U}_{a b}^{\{\sigma\}} \bar{c}_{a \sigma_{1}}^{\dagger} \bar{c}_{a \sigma_{2}} \bar{c}_{b \sigma_{3}}^{\dagger} \bar{c}_{b \sigma_{4}}
$$

where $\bar{t}_{a b}=\bar{t}_{a b}(I, I)$, and $\bar{U}_{a b}^{\{\sigma\}}=\bar{U}_{a b}^{\{\sigma\}}(I, I)$. The intercluster part is

$$
H^{\mathrm{ic}}=\sum_{I, J, a, b, \sigma} \delta \bar{t}_{a b}(I, J) \bar{c}_{a \sigma}^{\dagger}(I) \bar{c}_{b \sigma}(J),
$$

where $\delta \bar{t}_{a b}(I, J)=\bar{t}_{a b}(I, J)-\bar{t}_{a b}$. Here we have neglected the inter-cluster interactions. Originally, the CPT is formulated for local single-site interactions, thus the intercluster interactions are absent. Often quantum cluster approaches either neglect the inter-cluster interactions or treat them in a static mean-field approximation. However the inter-cluster interactions can be incorporated beyond the static mean-field approximation by employing a self consistent approach ${ }^{19,20}$ Note that the cluster hopping $\bar{t}_{a b}$ may couple any sites within the cluster. At a finite cluster it is reduced by a factor of $\mathcal{O}\left(1 / L_{c}^{2}\right)_{\underline{\underline{9}}}$ The inter-cluster hopping $\delta \bar{t} \sim 1 / L_{c}$ for large linear cluster size $L_{c}$ compared to its counterpart $t$ of the original $\mathrm{CPT}^{9.21}$ The nature of the approximation of the CPT is a strong coupling expansion in which the small parameter is the inter-cluster hopping ${ }^{15,16,17}$ Therefore in the PCPT the small parameter is truly $1 / L_{c}$.

Within the PCPT the cluster Hamiltonian (31) is solved exactly. We obtain the Dyson equation for the cluster Green function

$$
\bar{G}_{\overline{\mathbf{k}}}^{\mathrm{c}}(z)=\frac{1}{z-\bar{t}_{\overline{\mathbf{k}}}-\bar{\Sigma}_{\overline{\mathbf{k}}}^{\mathrm{c}}(z)},
$$

where $\bar{t}_{\overline{\mathbf{k}}}=\left(N_{c} / N\right) \sum_{\mathbf{K}} t(\mathbf{K}+\overline{\mathbf{k}})$, and $\bar{\Sigma}_{\overline{\mathbf{k}}}^{\mathrm{c}}(z)$ is the cluster self energy. Here the Dyson equation is written in the reciprocal space representation. We can make the Fourier transformation in the cluster space because the fermions are periodized in the cluster space, thus the cluster Green function and self energy are diagonal in the reciprocal space. As the CPT, within the PCPT the self energy of the lattice Green function is approximated by the cluster self energy. Thus we obtain

$$
\bar{G}_{\overline{\mathbf{k}}}(\mathbf{K}, z)=\frac{1}{z-\bar{t}_{\overline{\mathbf{k}}}(\mathbf{K})-\bar{\Sigma}_{\overline{\mathbf{k}}}^{c}(z)} .
$$

Since $\bar{G}_{\overline{\mathbf{k}}}(\mathbf{K}, z)=G(\mathbf{K}+\overline{\mathbf{k}}, z)$ and $\bar{t}_{\overline{\mathbf{k}}}(\mathbf{K})=t(\mathbf{K}+\overline{\mathbf{k}})$ we finally obtain the Green function of the original fermions

$$
G(\mathbf{K}+\overline{\mathbf{k}}, z)=\frac{1}{z-t(\mathbf{K}+\overline{\mathbf{k}})-\bar{\Sigma}_{\overline{\mathbf{k}}}^{c}(z)} .
$$

Equations (33)-(35) are the principal equations of the PCPT. As the CPT, the PCPT is exact in the limits $L_{c} \rightarrow \infty, U / t=0$, and $t / U=0$ for local interactions. The PCPT is formulated in the reciprocal space, hence it avoids the diagonalizations of the Green function in both real and reciprocal spaces, that significantly reduces the computational time compared to the CPT. Note that the present PCPT is different to the ordinary periodic $\mathrm{CPT}, \stackrel{17.18}{\longleftarrow}$ where the periodicity is taken into account by including additional hoppings. In the ordinary periodic CPT the hopping is still nearest neighbor, while in the present PCPT it is no longer only nearest neighbor. Moreover, in the present PCPT all hoppings within a cluster are treated exactly, whereas in the ordinary periodic CPT the adding and subtracting hoppings are treated on different footings.

For an illustration we study the one dimensional large$\mathcal{N}$ model, originally introduced by Affleck and Marston in two dimensions $\stackrel{22}{2}$ The model was also used for comparison between the DCA and CDMFT schemes ${ }^{12.13} \mathrm{We}$ will compare the PCPT and CPT schemes to its exact solution. The Hamiltonian of the model reads

$$
\begin{aligned}
H= & -t \sum_{<i, j>, \sigma} c_{i \sigma}^{\dagger} c_{j \sigma}+\mu \sum_{i \sigma} c_{i \sigma}^{\dagger} c_{i \sigma} \\
& +\frac{J}{2 \mathcal{N}} \sum_{<i, j>, \sigma, \sigma^{\prime}} c_{i \sigma}^{\dagger} c_{i \sigma^{\prime}} c_{j \sigma^{\prime}}^{\dagger} c_{j \sigma},
\end{aligned}
$$

where $\langle i, j\rangle$ denotes the nearest neighbor sites, and $\sigma=1, \ldots, \mathcal{N}$. As usually, $t$ is the hopping integral, $J$ is the exchange strength, and $\mu$ is the chemical potential. We will consider the large $\mathcal{N}$ limit. In this limit the model can be solved exactly. Indeed, the quantity $\chi=$ $(1 / \mathcal{N}) \sum_{\sigma} c_{i, \sigma}^{\dagger} c_{i+1, \sigma}$ does not fluctuate, that the static mean field theory is exact. We obtain the exact solution

$$
\begin{aligned}
\chi^{\text {exact }} & =\frac{1}{2 L} \sum_{\mathbf{k}} f(\beta E(\mathbf{k})) \gamma(\mathbf{k}), \\
E(\mathbf{k}) & =-\left(t+J \chi^{\text {exact }}\right) \gamma(\mathbf{k})+\mu,
\end{aligned}
$$




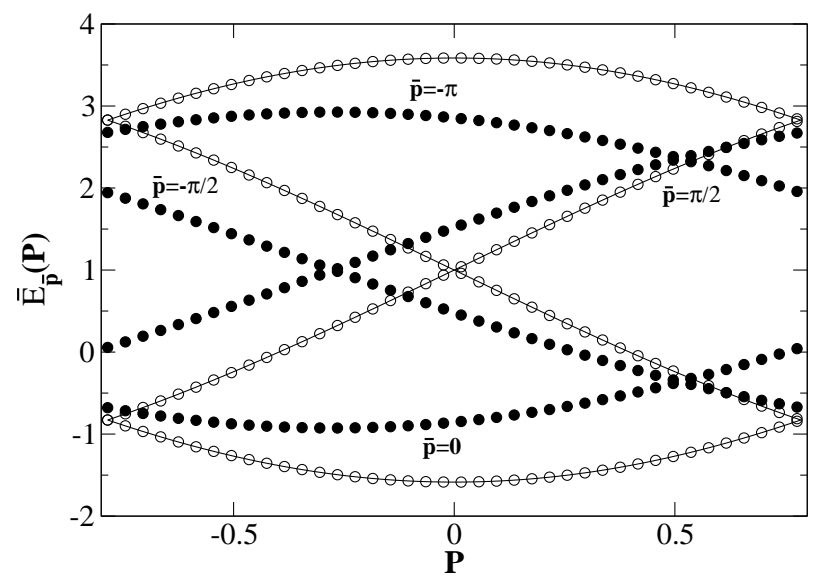

FIG. 1: The dispersion $\bar{E}_{\overline{\mathbf{p}}}(\mathbf{P})$ of the periodized fermions (the open symbols) for the cluster size $L_{c}=4$ in comparison with the dispersion $E(\mathbf{P}+\overline{\mathbf{p}})$ of the original fermions (the line). The filled symbols are $\bar{E}_{\overline{\mathbf{p}}}^{\mathrm{u}}(\mathbf{P})$ of the umklapp case for $L_{c}=4$. $(t=J=1, \mu=1, \beta=10)$.

where $\beta$ is the inverse temperature, and $f(x)=$ $1 /(\exp (x)+1)$ is the Fermi function, and $\gamma(\mathbf{k})=2 \cos (k)$.

Within the CPT, the cluster of size $L_{c}$ has the following Hamiltonian

$$
\begin{aligned}
H^{\mathrm{c}}= & -t \sum_{a=1}^{L_{c}-1} \sum_{\sigma} c_{a, \sigma}^{\dagger} c_{a+1, \sigma}+\text { h.c. }+\mu \sum_{a=1}^{L_{c}} \sum_{\sigma} c_{a \sigma}^{\dagger} c_{a \sigma} \\
& +\frac{J}{2 \mathcal{N}} \sum_{a=1}^{L_{c}-1} \sum_{\sigma, \sigma^{\prime}} c_{a, \sigma}^{\dagger} c_{a, \sigma^{\prime}} c_{a+1, \sigma^{\prime}}^{\dagger} c_{a+1, \sigma}+\text { h.c.. }
\end{aligned}
$$

The cluster Hamiltonian (39) can be solved exactly, for instance by the exact diagonalization. However, at the large- $\mathcal{N}$ limit it can simply be solved exactly. Indeed, the cluster quantity $\chi_{a}^{\mathrm{c}}=(1 / \mathcal{N}) \sum_{\sigma} c_{a, \sigma}^{\dagger} c_{a+1, \sigma}$ does not fluctuate, and the cluster Hamiltonian (39) becomes quadratic and can simply be diagonalized. However, in this case $\chi_{a}^{\mathrm{c}}$ may depends on the cluster index. The cluster Green function is

$$
\hat{G}^{\mathrm{c}}(z)=\left[(z-\mu) \hat{I}-\hat{t}-\hat{\Sigma}^{\mathrm{c}}\right]^{-1},
$$

where $\hat{t}$ is the cluster hopping matrix, and $\hat{\Sigma}^{c}$ is the cluster self energy

$$
\Sigma_{a b}^{\mathrm{c}}=-J\left(\chi_{a}^{\mathrm{c}} \delta_{a, b-1}+\chi_{b}^{\mathrm{c}} \delta_{a, b+1}\right) .
$$

The cluster quantity $\chi_{a}^{c}$ can be calculated from the cluster Green function

$$
\chi_{a}^{c}=-\int \frac{d \omega}{\pi} f(\beta \omega) \operatorname{Im} G_{a+1, a}^{c}\left(\omega+i 0^{+}\right) .
$$

Equations (40)-(42) give the exact solution of the cluster Hamiltonian (39) at the large- $\mathcal{N}$ limit. Once the exact

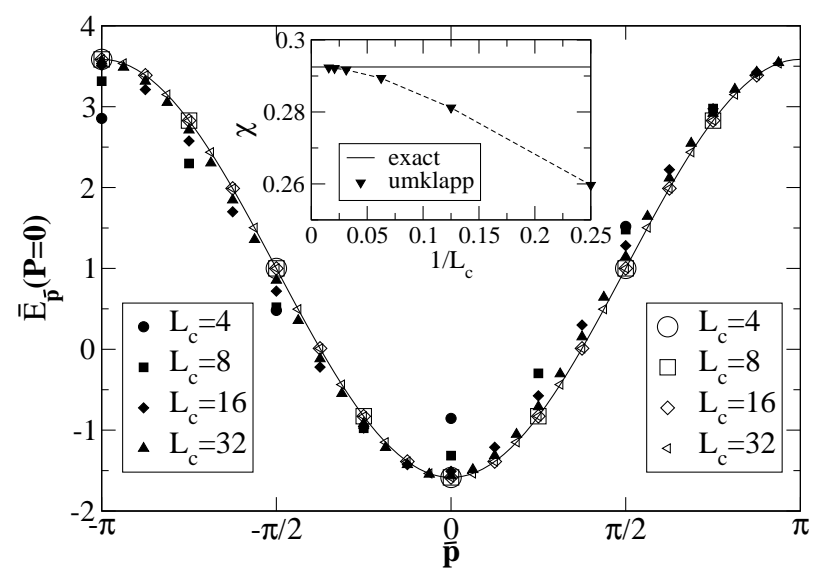

FIG. 2: The dispersion $\bar{E}_{\overline{\mathbf{p}}}(\mathbf{P}=0)$ of the periodized fermions for various cluster sizes $L_{c}$ (the open symbols) in comparison with $E(\mathbf{P}+\overline{\mathbf{p}})$ of the original fermions (the line) at $\mathbf{P}=0$. The filled symbols are $\bar{E}_{\overline{\mathbf{p}}}^{\mathrm{u}}(\mathbf{P}=0)$ of the umklapp case. The inset plots $\chi^{\mathrm{u}}$ of the umklapp case in comparison with the exact value. $(t=J=1, \mu=1, \beta=10)$.

cluster solution is obtained, the lattice Green function within the CPT is computed by ${ }^{16,17}$

$$
G^{\mathrm{CPT}}(\mathbf{k}, z)=\frac{1}{L_{c}} \sum_{a b} G_{a b}(\mathbf{K}, z) e^{-i \mathbf{k} \cdot\left(\overline{\mathbf{r}}_{a}-\overline{\mathbf{r}}_{b}\right)},
$$

where $G_{a b}^{-1}(\mathbf{K}, z)=(z-\mu) \delta_{a b}-t_{a b}(\mathbf{K})-\Sigma_{a b}^{\mathrm{c}}$. The lattice quantity $\chi=(1 / \mathcal{N}) \sum_{\sigma} c_{i, \sigma}^{\dagger} c_{i+1, \sigma}$ within the CPT is calculated from this lattice Green function

$$
\begin{gathered}
\chi^{\mathrm{CPT}}=-\int \frac{d \omega}{\pi} f(\beta \omega) \operatorname{Im} G_{i+1, i}^{\mathrm{CPT}}\left(\omega+i 0^{+}\right) \\
=-\frac{1}{L} \sum_{\mathbf{K}, a} \int \frac{d \omega}{\pi} f(\beta \omega) \operatorname{Im} G_{a+1, a}\left(\mathbf{K}, \omega+i 0^{+}\right) .
\end{gathered}
$$

Next, we consider the one dimensional large- $\mathcal{N}$ model (36) in the periodization form. The periodized Hamiltonian reads

$$
\begin{aligned}
H & =\sum_{I, J, a, b, \sigma} \bar{t}_{a b}(I, J) \bar{c}_{a \sigma}^{\dagger}(I) \bar{c}_{b \sigma}(J)+\mu \sum_{I, a, \sigma} \bar{c}_{a \sigma}^{\dagger}(I) \bar{c}_{a \sigma}(I) \\
& +\frac{1}{2 \mathcal{N}} \sum_{I, J, a, b, \sigma, \sigma^{\prime}} \bar{J}_{a b}(I, J) \bar{c}_{a \sigma}^{\dagger}(I) \bar{c}_{a \sigma^{\prime}}(I) \bar{c}_{b \sigma^{\prime}}^{\dagger}(J) \bar{c}_{b \sigma}(J),(45)
\end{aligned}
$$

where $\bar{t}_{a b}(I, J)$ and $\bar{J}_{a b}(I, J)$ are determined by the periodization transformation (18) and (19), respectively. The Hamiltonian (45) can be solved exactly in the large- $\mathcal{N}$ limit. Introducing quantity $\bar{\chi}_{\overline{\mathbf{p}}}(\mathbf{P})=$ $(1 / \mathcal{N}) \sum_{\sigma} \bar{c}_{\overline{\mathbf{p}} \sigma}^{\dagger}(\mathbf{P}) \bar{c}_{\overline{\mathbf{p}} \sigma}(\mathbf{P})$ we obtain

$$
\begin{aligned}
\bar{\chi}_{\overline{\mathbf{p}}}(\mathbf{P})= & f\left(\beta \bar{E}_{\overline{\mathbf{p}}}(\mathbf{P})\right) \\
\bar{E}_{\overline{\mathbf{p}}}(\mathbf{P})= & -t \gamma(\mathbf{P}+\overline{\mathbf{p}})+\mu \\
& -J \frac{1}{L} \sum_{\mathbf{K}, \overline{\mathbf{k}}} \gamma(\mathbf{P}+\overline{\mathbf{p}}-\mathbf{K}-\overline{\mathbf{k}}) \bar{\chi}_{\overline{\mathbf{k}}}(\mathbf{K})
\end{aligned}
$$


When Eqs. (46)-(47) are solved, the quantity $\chi=$ $(1 / \mathcal{N}) \sum_{\sigma} c_{i, \sigma}^{\dagger} c_{i+1, \sigma}$ of the original fermions can be calculated too

$$
\chi^{\mathrm{P}}=\frac{1}{2 L} \sum_{\mathbf{P}, \overline{\mathbf{p}}} f\left(\beta \bar{E}_{\overline{\mathbf{p}}}(\mathbf{P})\right) \gamma(\mathbf{P}+\overline{\mathbf{p}}) .
$$

Numerically, we alway obtain $\chi^{\mathrm{P}}=\chi^{\text {exact }}$ independently on the cluster size $L_{c}$. Moreover, since the Green function $\ll \bar{c}_{\overline{\mathbf{k}}}(\mathbf{K})\left|\bar{c}_{\overline{\mathbf{k}}}^{\dagger}(\mathbf{K}) \gg=\ll c(\mathbf{K}+\overline{\mathbf{k}})\right| c^{\dagger}(\mathbf{K}+\overline{\mathbf{k}}) \gg$, one must have the identity of the dispersions of periodized and original fermions $\bar{E}_{\overline{\mathbf{p}}}(\mathbf{P})=E(\mathbf{P}+\overline{\mathbf{p}})$. In Fig. 1 we plot the dispersions for the cluster size $L_{c}=4$. It confirms the identity of the dispersions of periodized and original fermions. In Fig. 2 we plot the dispersions at fixed $\mathbf{P}=0$ for various cluster sizes $L_{c}$. It shows the identity of the dispersions of periodized and original fermions is independent on the cluster size. These results confirm the equivalent of the periodized and original Hamiltonians. Next, we consider the umklapp case where the constrain (16) is relaxed by its exact relation. Then we obtain

$$
\begin{aligned}
\bar{\chi}_{\overline{\mathbf{p}}}^{\mathrm{u}}(\mathbf{P}) & =f\left(\beta \bar{E}_{\overline{\mathbf{p}}}^{\mathrm{u}}(\mathbf{P})\right), \\
\bar{E}_{\overline{\mathbf{p}}}^{\mathrm{u}}(\mathbf{P}) & =-t \gamma(\mathbf{P}+\overline{\mathbf{p}})+\mu \\
-J & \frac{1}{L} \sum_{\mathbf{K}, \overline{\mathbf{k}}} \gamma(\mathbf{P}+\overline{\mathbf{p}}-\mathbf{K}-\overline{\mathbf{k}}+\overline{\mathbf{G}}) \bar{\chi}_{\overline{\mathbf{k}}}^{\mathrm{u}}(\mathbf{K}),
\end{aligned}
$$

where $\overline{\mathbf{G}}$ is the umklapp transfer momentum. Since $|\overline{\mathbf{G}}| \sim 1 / L_{c}$, in the large $L_{c}$ limit this umklapp case approaches to the normal one. The lattice quantity $\chi=(1 / \mathcal{N}) \sum_{\sigma} c_{i, \sigma}^{\dagger} c_{i+1, \sigma}$ of the original fermions can be calculated by

$$
\chi^{\mathrm{u}}=\frac{1}{2 L} \sum_{\mathbf{P}, \overline{\mathbf{p}}} f\left(\beta \bar{E}_{\overline{\mathbf{p}}}^{\mathrm{u}}(\mathbf{P})\right) \gamma(\mathbf{P}+\overline{\mathbf{p}}) .
$$

In numerical calculations we take $\overline{\mathbf{G}}=2 \pi / L_{c}$. It turns out numerically that $\chi^{\mathrm{u}}$ is never equal to $\chi^{\text {exact }}$, although it approaches to the exact value as $1 / L_{c}^{2}$, as shown in the inset of Fig. 2 In Fig. 1 and 2 we plot also the dispersion $\bar{E}_{\overline{\mathbf{p}}}^{\mathrm{u}}(\mathbf{P})$. These figures show that the dispersion of the periodized fermions in the presence of the umklapp momentum transfer is not exact. These results confirm that the constrain is important in order to maintain the correct physics.

Now we apply the PCPT to the one dimensional large$\mathcal{N}$ model (36). The cluster periodized Hamiltonian is obtained from 45.

$$
\begin{aligned}
H^{c}= & -t \sum_{a, b, \sigma} \bar{\gamma}_{a b} \bar{c}_{a \sigma}^{\dagger} \bar{c}_{b \sigma}+\mu \sum_{a, \sigma} \bar{c}_{a \sigma}^{\dagger} \bar{c}_{a \sigma} \\
& +\frac{J}{2 \mathcal{N}} \sum_{a, b, \sigma, \sigma^{\prime}} \bar{\gamma}_{a b} \bar{c}_{a \sigma}^{\dagger} \bar{c}_{a \sigma^{\prime}} \bar{c}_{b \sigma^{\prime}}^{\dagger} \bar{c}_{b \sigma} .
\end{aligned}
$$

At the large- $\mathcal{N}$ limit the cluster Hamiltonian (52) can simply be solved exactly. Introducing the cluster quantity $\bar{\chi}_{\overline{\mathbf{k}}}^{\mathrm{c}}=(1 / \mathcal{N}) \sum_{\sigma} \bar{c}_{\overline{\mathbf{k}} \sigma}^{\dagger} \bar{c}_{\overline{\mathbf{k}} \sigma}$ we obtain the cluster self

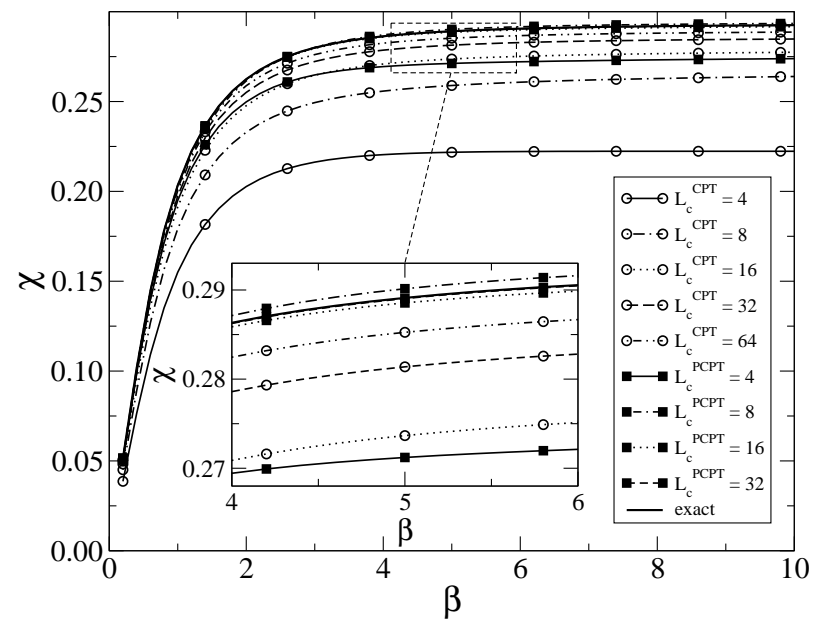

FIG. 3: The lattice quantity $\chi$ as a function of inverse temperature $\beta$ for the CPT and PCPT with different cluster sizes $L_{c}(t=J=1, \mu=1)$.

energy

$$
\bar{\Sigma}_{\overline{\mathbf{k}}}^{\mathrm{c}}=-J \frac{1}{L_{c}} \sum_{\overline{\mathbf{p}}} \bar{\gamma}_{\overline{\mathbf{k}}-\overline{\mathbf{p}}} \bar{\chi}_{\overline{\mathbf{p}}}^{\mathrm{c}}
$$

where $\bar{\gamma}_{\overline{\mathbf{k}}}=\left(L_{c} / L\right) \sum_{\mathbf{K}} \gamma(\mathbf{K}+\overline{\mathbf{k}})$. The cluster quantity $\bar{\chi}_{\overline{\mathbf{k}}}^{\mathrm{c}}$ can be calculated from the cluster Green function. We obtain

$$
\begin{aligned}
& \bar{\chi}_{\overline{\mathbf{k}}}^{\mathrm{c}}=f\left(\beta \bar{E}_{\overline{\mathbf{k}}}^{\mathrm{c}}\right), \\
& \bar{E}_{\overline{\mathbf{k}}}^{\mathrm{c}}=-t \bar{\gamma}_{\overline{\mathbf{k}}}+\mu-J \frac{1}{L_{c}} \sum_{\overline{\mathbf{p}}} \bar{\gamma}_{\overline{\mathbf{k}}-\overline{\mathbf{p}}} \bar{\chi}_{\overline{\mathbf{p}}}^{\mathrm{c}} .
\end{aligned}
$$

Finally, once the cluster equations (54)-(155) are solved, we obtain the lattice Green function of the original fermions

$$
G^{\mathrm{PCPT}}(\mathbf{k}, z)=\bar{G}_{\overline{\mathbf{k}}}(\mathbf{K}, z)=\frac{1}{z+t \gamma(\mathbf{k})-\mu-\bar{\Sigma}_{\overline{\mathbf{k}}}^{\mathrm{c}}} .
$$

The lattice quantity $\chi^{\mathrm{PCPT}}=(1 / \mathcal{N}) \sum_{\sigma} c_{i, \sigma}^{\dagger} c_{i+1, \sigma}$ is calculated from this lattice Green function. We obtain

$$
\begin{aligned}
\chi^{\mathrm{PCPT}} & =\frac{1}{2 L} \sum_{\mathbf{k}} f\left(\beta E^{\mathrm{PCPT}}(\mathbf{k})\right) \gamma(\mathbf{k}), \\
E^{\mathrm{PCPT}}(\mathbf{k}) & =-t \gamma(\mathbf{k})+\mu+\bar{\Sigma}_{\overline{\mathbf{k}}}^{\mathrm{c}} .
\end{aligned}
$$

So far we have obtained the quantity $\chi$ in both the CPT and PCPT, as well as its exact value. We use it to compare the error and the convergence of the $\mathrm{CPT}$ and PCPT schemes.

In Fig. 3 we plot the lattice quantity $\chi$ as a function of inverse temperature calculated within the $\mathrm{CPT}$ and the PCPT. It is clear that both the CPT and PCPT results converge well to the exact solution. The CPT 
results converge consistently, whereas for small cluster sizes the PCPT results fluctuate around the exact solution. However, the PCPT results converge faster than the CPT ones. Even at small cluster sizes the PCPT results are closer to the exact solution than the CPT ones. In Fig. 4 we plot $\chi$ as a function of $1 / L_{c}$ at fixed temperature. It shows that the CPT results converge linearly in $1 / L_{c}$, whereas the PCPT results converge quadratically in $1 / L_{c}$. The convergence features of the CPT and PCPT are quite similar to the ones of the CDMFT and DCA $9.12,13$ The convergence can be understood in the term of the hybridization in the locator expansion. 9 Indeed, the convergence of $\chi$ depends mostly on the convergence of the coarse grained Green function (see Eq. (44))

$$
\hat{G}(z)=\frac{L_{c}}{L} \sum_{\mathbf{K}} \hat{G}(\mathbf{K}, z),
$$

where the Green function $\hat{G}(\mathbf{K}, z)$ can be rewritten as

$$
\hat{G}(\mathbf{K}, z)=\left[\left[\hat{G}^{\mathrm{c}}(z)\right]^{-1}-\delta \hat{t}(\mathbf{K})\right]^{-1} .
$$

This is also valid for the PCPT just by replacing the coarse grained Green function of the original fermions by its counterpart of the periodized fermions. The coarse grained Green function can be rewritten in the locator expansion as 9

$$
\hat{G}(z)=\left[\left[\hat{G}^{\mathrm{c}}(z)\right]^{-1}-\hat{\Gamma}(z)\right]^{-1}
$$

where $\hat{\Gamma}(z)$ is the hybridization function

$$
\begin{aligned}
\hat{\Gamma}(z)= & {\left[\hat{I}+\frac{L_{c}}{L} \sum_{\mathbf{K}} \delta \hat{t}(\mathbf{K}) \hat{G}(\mathbf{K}, z)\right]^{-1} } \\
& \frac{L_{c}}{L} \sum_{\mathbf{K}} \delta \hat{t}(\mathbf{K}) \hat{G}(\mathbf{K}, z) \delta \hat{t}(\mathbf{K}) .
\end{aligned}
$$

The hybridization is an amplitude for fermion hopping from a cluster into the surrounding and back again into the cluster. It acts as the small parameter in the expansion of the coarse grained Green function (61). As in the DCA, the inter-cluster hopping $\delta \bar{t}$ in the PCPT scales like $1 / L_{c}$ for large cluster sizes, whereas in the CPT and the CDMFT it is of order 1 . The average hybridization per cluster site scales like $1 / L_{c}^{2}$ in the PCPT, and like $1 / L_{c}$ in the $\mathrm{CPT}^{9}$ Therefore the PCPT converges like $1 / L_{c}^{2}$, whereas the CPT converges like $1 / L_{c}$. Note that these convergences are valid for the coarse grained Green function in any dimensions. The convergence feature is an advantage of the PCPT in comparison with the CPT.

\section{CONCLUSION}

We have introduced a canonical transformation which periodizes fermions in the cluster space. By applying the canonical transformation to the Hamiltonian, the peri-

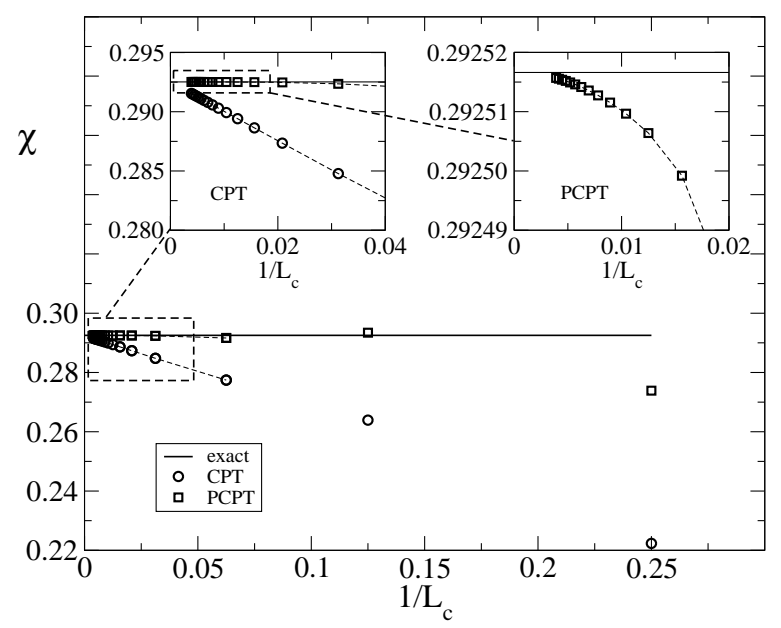

FIG. 4: The lattice quantity $\chi$ as a function of $1 / L_{c}$ for the $\mathrm{CPT}$ and PCPT at fixed temperature $\beta=10(t=J=1$, $\mu=1)$.

from the superlattice to the cluster space. Within the periodized Hamiltonian, the DCA can be derived from the CDMFT. In the such way, the DCA and the CDMFT can be unified into a single microscopic definition. It also gives an alternative microscopic background of the discretization of irreducible quantities on the reciprocal space, and clarifies the approximation nature of the DCA. We also develop the PCPT in which the wave vector within a cluster is a conserved quantum number. This allows to avoid the matrix inversion of the Green function that reduces significantly the computation time when the cluster size is large. The PCPT can work on both the direct and reciprocal spaces. As the CPT, the PCPT is exact in the limits $L_{c} \rightarrow \infty, U / t=0$, and $t / U=0$. It is also clarified that the small parameter of the PCPT is $1 / L_{c}$. As a benchmark the exact one dimension $1 / \mathcal{N}$ model is studied. It turns out that the PCPT converges rapidly with corrections $\mathcal{O}\left(1 / L_{c}^{2}\right)$, whereas the standard CPT converges with corrections $\mathcal{O}\left(1 / L_{c}\right)$.

\section{Acknowledgments}

The author would like to thank Professor S.W. Kim and the Asia Pacific Center for Theoretical Physics for the hospitality. He also acknowledges the Department of Physics, POSTECH for sharing CPU's time. This work was supported by the Asia Pacific Center for Theoretical Physics. 
1 W. Metzner and D. Vollhardt, Phys. Rev. Lett. 62, 324 (1989).

2 A. Georges, G. Kotliar, W. Krauth, and M.J. Rozenberg, Rev. Mod. Phys. 68, 13 (1996).

3 P.L. Leath and B. Goodman, Phys. Rev. 148, 968 (1966).

4 P. Soven, Phys. Rev. 156, 809 (1967).

${ }^{5}$ D.W. Taylor, Phys. Rev. 156, 1017 (1967).

${ }^{6}$ F. Ducastelle, J. Phys. C: Sol. State Phys. 7, 1795 (1974).

7 G. Kotliar, S.Y. Savrasov, G. Palsson, and G. Biroli, Phys. Rev. Lett. 87, 186401 (2001).

8 M.H. Hettler, A.N. Tahvildar-Zadeh, and M. Jarrell, T. Pruschke, and H.R. Krishnamurthy, Phys. Rev. B 58, R7475 (1998).

9 T. Maier, M. Jarrell, T. Pruschke, and M. H. Hettler, Rev. Mod. Phys. 77, 1027 (2005).

10 M. Potthoff, Eur. Phys. J. B 32, 429 (2003); 36, 335 (2003).

11 M. Potthoff, M. Aichhorn, and C. Dahnken, Phys. Rev. Lett. 91, 206402 (2003).

12 G. Biroli and G. Kotliar, Phys. Rev. B 65, 155112 (2002);
71, 037102 (2005).

13 K. Aryanpour, Th.A. Maier, and M. Jarrell, Phys. Rev. B 71, 037101 (2005).

14 G. Biroli, O. Parcollet, and G. Kotliar, Phys. Rev. B 69, 205108 (2004).

15 C. Gros and R. Valenti, Phys. Rev. B 48, 418 (1993).

16 D. Senechal, D. Perez, and M. Pioro-Ladriere, Phys. Rev. Lett. 84, 522 (2000).

17 D. Senechal, D. Perez, and D. Plouffe, Phys. Rev. B 66, 075129 (2002).

18 C. Dahnken, E. Arrigoni, and W. Hanke, J. Low Temp. Phys. 126, 949 (2002).

19 J.L. Smith and Q. Si, Phys. Rev. B 61, 5184 (2000).

20 Th. A. Maier, Physica B 359-361, 512 (2005).

21 Th. Maier, M. Jarrell, Th. Pruschke, and J. Keller, Eur. Phys. J B 13, 613 (2000).

22 I. Affleck and J.B. Marston, Phys. Rev. B 37, R3774 (1988). 